\title{
改进液相氧化还原法制备高性能氢气吸附用石墨烯
}

\author{
袁文辉 ${ }^{1, *} \quad$ 李保庆 ${ }^{1} \quad$ 李 莉 $^{2}$ \\ ('华南理工大学化学与化工学院, 广州 510640; 2华南理工大学环境科学与工程学院, 广州 510640)
}

\begin{abstract}
摘要: 以液相氧化还原法为基础, 并在分散剂十二烷基苯磺酸钠(SDBS)作用下制备得到高质量石墨烯, 有效 避免了在此过程中石墨烯大量团聚的现象. 采用X射线衍射 $(X R D)$ 、拉曼光谱(RS)、扫描电子显微镜(SEM)、透 射电子显微镜(TEM)和原子力显微镜(AFM)等分析手段对石墨烯样品进行了表征. XRD 结果体现了石墨、氧化 石墨和石墨烯晶型结构的区别; SEM 和 TEM 结果显示石墨烯呈网格状, 表面平整, 缺陷少; AFM 分析表明样品 中单层石墨烯厚度约为 $1.3 \mathrm{~nm}$, 同时也存在少许双层结构. BET测试法得到石墨烯的比表面积高达 $1206 \mathrm{~m}^{2}$. $\mathrm{g}^{-1}$, 考察了石墨烯在高压条件下对 $\mathrm{H}_{2}$ 的吸附性能. 通过对方法改进前后所制备的石墨烯样品进行比较, 结果表 明, 十二烷基苯磺酸钠的加入有效地减小了石墨烯的大量团聚, 且得到了高质量的石墨烯. 在 25 和 $55^{\circ} \mathrm{C}$ 条件 下, 高质量石墨烯对氢气的吸附量分别达到 $1.7 \%(w)$ 和 $1.1 \%(w)$, 比之前研究结果有了很大提高.
\end{abstract}

关键词：石墨烯；氧化石墨；氢气吸附；氧化还原；超声剥离 中图分类号: 0647

\section{Superior Graphene for Hydrogen Adsorption Prepared by the Improved Liquid Oxidation-Reduction Method}

\author{
YUAN Wen-Hui ${ }^{1, *} \quad$ LI Bao-Qing ${ }^{1} \quad$ LI Li $^{2}$ \\ ('School of Chemistry and Chemical Engineering, South China University of Technology, Guangzhou 510640, P. R. China; \\ ${ }^{2}$ College of Environmental Science and Engineering, South China University of Technology, Guangzhou 510640, P. R. China)
}

\begin{abstract}
Graphite oxide (GO) was prepared from liquid oxidation based on Hummers method and the graphene was then prepared using sodium borohydride to reduce the exfoliated graphite oxide by ultrasonication during which moderate sodium dodecyl benzene sulfonate (SDBS) was added into the suspension to reduce the agglomeration among the graphene layers and to obtain a stable graphene suspension. The as-prepared graphene was characterized by X-ray diffraction (XRD), Raman spectroscopy (RS), scanning electron microscopy (SEM), transmission electron microscopy (TEM), and atomic force microscopy (AFM). XRD results show that the crystal structures are different among graphite, graphite oxide, and graphene. SEM and TEM images show that graphene possesses a gridding structure, a smooth surface, and few defects. AFM analysis indicates that the thickness of the single layer graphene is about $1.3 \mathrm{~nm}$ while there are still a few double layers in the sample. The BET specific surface area of the graphene was about $1206 \mathrm{~m}^{2} \cdot \mathrm{g}^{-1}$ and its $\mathrm{H}_{2}$ adsorption properties were investigated under high pressure. The samples prepared by liquid oxidation-reduction were compared with that prepared by the improved liquid oxidation-reduction method, which indicates that the addition of SDBS effectively reduces agglomeration among the graphene layers and this generates high quality graphene. The adsorption capacities of $\mathrm{H}_{2}$ on graphene at 25 and $55^{\circ} \mathrm{C}$ reached $1.7 \%(w)$ and $1.1 \%(w)$, respectively, which are much higher than that reported previously.
\end{abstract}

Received: April 7, 2011; Revised: May 18, 2011; Published on Web: June 28, 2011.

"Corresponding author. Email: cewhyuan@scut.edu.cn; Tel: +86-20-87111887.

The project was supported by the National Natural Science Foundation of China (20976057).

国家自然科学基金(20976057)资助项目

(C) Editorial office of Acta Physico-Chimica Sinica 
Key Words: Graphene; Graphite oxide; Hydrogen adsorption; Oxidation-reduction; Ultrasonic exfoliated

\section{1 引言}

当前的能源生产与消费方式使人类的生态环 境遭到了严重破坏, 而且不断恶化. 因此, 寻求新的 替代型清洁能源已是迫在眉睫. 氢能由于具有清 洁、高效、可再生等优点被誉为 21 世纪理想能源, ${ }^{1-3}$ 而且越来越受到各国科学家的重视. 如何高效、简 单地存储氢气并达到美国能源部提出的 $6.0 \%(w)$ 吸 附量的目标已是当今备受关注的问题.

纳米碳材料具有原子质量低、化学稳定性好以 及丰富的孔道结构等优点, 近年来被广泛应用于氢 气存储领域. ${ }^{4-9}$ 石墨烯是近年来碳族材料中发现的 最新同素异形体, 它是由碳原子紧密堆积而成的单 层二维蜂窝状晶格结构的晶体薄膜, 其厚度只有 $0.335 \mathrm{~nm} .{ }^{10}$ 由于具有高的比表面积(约 $2630 \mathrm{~m}^{2} \cdot \mathrm{g}^{-1}$ )、 丰富的孔道结构、优良的导电率和易改性的表面化 学 ${ }^{11-15}$ 等特性, 自从 2004 年被英国 Manchester 大学 的 Geim 小组 ${ }^{11}$ 发现以来, 石墨烯就被广泛应用于气 体传感器、锂离子电池、晶体管、氢气存储、催化剂 等领域. ${ }^{16-19}$ 但是石墨烯的大量制备仍然面临着挑 战, 主要在于液相还原氧化石墨过程中石墨烯会不 可避免地发生团聚, 导致得不到高质量的单层石墨 烯, 同时这也阻碍了石墨烯在吸附领域的应用. 因 此, 寻找有效且能够大量制备单层石墨烯的方法成 为当前研究的热点.

Srinivas 等 ${ }^{20}$ 用 Hummers 方法氧化石墨, 在水中 超声剥离形成氧化石墨烯后, 再用水合肼还原制备 石墨烯, 在压力为 $1000 \mathrm{kPa}$, 温度为 -196 和 $25^{\circ} \mathrm{C}$ 下, 氢气的吸附量分别达到 $1.2 \%(w)$ 和 $0.1 \%(w)$. Ghosh 等 ${ }^{21}$ 使氧化石墨在高温下热剥离制备石墨烯, 在 $10000 \mathrm{kPa}$ 和 $65^{\circ} \mathrm{C}$ 条件下, 测得氢气吸附量约 $2.0 \%$ $(w)-3.1 \%(w)$. Cheng 等 ${ }^{22}$ 的研究结果表明在 100 $\mathrm{kPa} 、-196{ }^{\circ} \mathrm{C}$ 和 $6000 \mathrm{kPa} 、 25^{\circ} \mathrm{C}$ 条件下, 石墨烯对氢 气的吸附量分别达到 $0.4 \%(w)$ 和 $0.2 \%(w)$. 另外, 大 量研究 ${ }^{16,20-24}$ 制备的石墨烯因团聚而导致其石墨烯 比表面积远远小于理论比表面积, 达不到美国能源 部提出的 $6.0 \%(w)$ 的氢气吸附量指标. 因此, 如何得 到稳定的石墨烯悬浮液制备高质量单层石墨烯来 提高氢气吸附量成为石墨烯在能源领域研究的重 要目标. 近年来, Lotya ${ }^{25}$ 和 $\mathrm{Xu}^{26}$ 等分别用胆酸钠和吡 啶酸作为稳定剂制备了石墨烯悬浮液, 显示了表面
活性剂在制备高质量石墨烯中的优良作用.

本文在分散剂十二烷基苯磺酸钠(SDBS)作用 下, 以氧化石墨为原料, 通过 $\mathrm{NaBH}_{4}$ 液相还原法制 备得到了高质量石墨烯. 采用XRD、Raman、SEM、 TEM 和 AFM 等分析手段对石墨烯样品进行了详细 表征, BET 测试法证明了石墨烯的高比表面积和丰 富的孔道结构, 用氢气高压吸附实验验证了石墨烯 作为一种多孔吸附材料的可观前景.

\section{2 实 验}

\section{1 试剂与仪器}

石 墨 (约 325 mesh; $45 \mu \mathrm{m} ; 99.8 \%$ ), 高锰酸钾 (AR, 99.5\%), 高氯酸钾 (AR, $\geq 99.5 \%)$, 浓硫酸 $(A R$, $95.0 \%-98.0 \%)$, 硝酸钠 (AR, $99.0 \%)$, 嗍氢化钠 (AR, $\geq 96.0 \%$ ) 等试剂均购于 Alfa Aesar(北京), 十二 烷基苯磺酸钠 $(\mathrm{CR}, \geq 85 \%)$, 双氧水( $\mathrm{AR}, 30 \%)$, 盐酸 (AR, 36\%-38\%), 丙酮(AR, 99.5\%)等购于国药集团 化学试剂有限公司, 所有溶液均用高纯水配制.

科大创新股份有限公司中佳分公司 HC-3518 高速离心机; 德国 Bruker 公司 XRD 射线衍射仪; 日 本 Hitachi 公司 S-3700N 型扫描电子显微镜; 荷兰 Philips-FEI 公司 Tecnai G2 F30 S-Twin 型透射电子 显微镜; 美国 Veeco Multimode 3D 原子力显微镜; 美 国 Micromeritics ASAP 2010 比表面积分析仪; 德国 Rubotherm 磁悬浮天平.

\section{2 氧化石墨的制备}

采用 Hummers 法 ${ }^{27}$ 制备氧化石墨. 在冰水浴中 装配好 $500 \mathrm{~mL}$ 的反应瓶, 将 $5 \mathrm{~g}$ 石墨粉和 $5 \mathrm{~g}$ 硝酸钠 与 $200 \mathrm{~mL}$ 浓硫酸混合均匀, 搅拌下加入 $25 \mathrm{~g}$ 高氯酸 钾, 均匀后, 再分数次加入 $15 \mathrm{~g}$ 高锰酸钾, 控制温度 不超过 $20^{\circ} \mathrm{C}$, 搅拌一段时间后, 撤去冰浴, 将反应瓶 转移至电磁搅拌器上, 电磁搅拌持续 $24 \mathrm{~h}$ 之后, 摚 拌下缓慢加入 $200 \mathrm{~mL}$ 去离子水, 温度升高到 $98^{\circ} \mathrm{C}$ 左右, 摚拌 $20 \mathrm{~min}$ 后, 加入适量双氧水还原残留的 氧化剂, 使溶液变为亮黄色. 然后分次以 $10000 \mathrm{r}$. $\mathrm{min}^{-1}$ 转速离心分离氧化石墨悬浮液, 并先后用 $5 \%$ $\mathrm{HCl}$ 溶液和去离子水洗涤直到分离液 $\mathrm{pH}=7$. 将得到 的滤饼真空干燥即得氧化石墨.

\section{3 石墨烯的制备}

将氧化石墨研碎, 称取 $300 \mathrm{mg}$ 分散于 $60 \mathrm{~mL}$ 去 
离子水中, 得到棕黄色的悬浮液, 超声分散 $1 \mathrm{~h}$ 后得 到稳定的胶状悬浮液. 然后转移到四口烧瓶中, 加 入 $600 \mathrm{mg}$ 硼氢化钠和 $50 \mathrm{mg}$ 十二烷基苯磺酸钠, 升 温至 $80^{\circ} \mathrm{C}$, 在此条件下回流 $16 \mathrm{~h}$ 后离心分离, 依次 用丙酮和去离子水洗涤至 $\mathrm{pH}=7$, 将得到的滤饼真空 干燥后保存备用, 记为 $\mathrm{GS}_{1}$. 按照同样的方法在不加 分散剂的条件下制备得到石墨烯 $\mathrm{GS}_{2}$.

\section{4 测试与表征}

采用 X射线衍射(XRD)仪对石墨、氧化石墨、石 墨烯的晶相结构进行分析, 测试条件为: $\mathrm{Cu}$ 靶 $K_{a}$, $\lambda=1.54 \times 10^{-10} \mathrm{~m}$ 线作射线源, 管电流 $30 \mathrm{~mA}$, 管电压 $40 \mathrm{kV}$, 扫描范围 $5^{\circ}-60^{\circ}$. 采用扫描电子显微镜 (SEM) 观察石墨烯表面形貌和结构. 样品表面喷涂 金膜, 镀金时间为 $5 \mathrm{~min}$. 采用透射电子显微镜 (TEM) 观察石墨烯表面超微结构. 采用原子力显微 镜(AFM)观察石墨烯微观表面并确定其层数. 以水 作为分散介质, 配制 $1 \mathrm{mg} \cdot \mathrm{L}^{-1}$ 的石墨烯悬浮液, 以云 母片为基底. 采用比表面积分析仪在液氮温度下得 到样品的氮气吸附-脱附等温线和孔径分布(实验前 样品在 $150^{\circ} \mathrm{C}$ 下处理 $5 \mathrm{~h}$ ).

\section{5 氢气的高压吸附性能测试}

氢气的高压吸附性能测试 $(0-3000 \mathrm{kPa})$ 是采用 重量法在德国 Rubotherm 磁悬浮天平上进行的. 该 天平配有自动气体进样系统、压力控制系统和温度 控制系统. 实验中吹扫气为高纯氦气( $99.999 \%)$, 吸 附气为高纯氢气 $(99.999 \%)$. 具体的实验步骤为: 先 称量 1/3-1/2 样品框体积的样品置于不锈钢样品框 中, 在 $150{ }^{\circ} \mathrm{C}$ 条件下抽真空预处理 $12 \mathrm{~h}$. 之后开始进 行吸附实验, 调节质量流量控制器(MFC)分别控制 吸附气和吹扫气的流速, 使其均为 $30 \mathrm{~mL} \cdot \mathrm{min}^{-1}$, 调

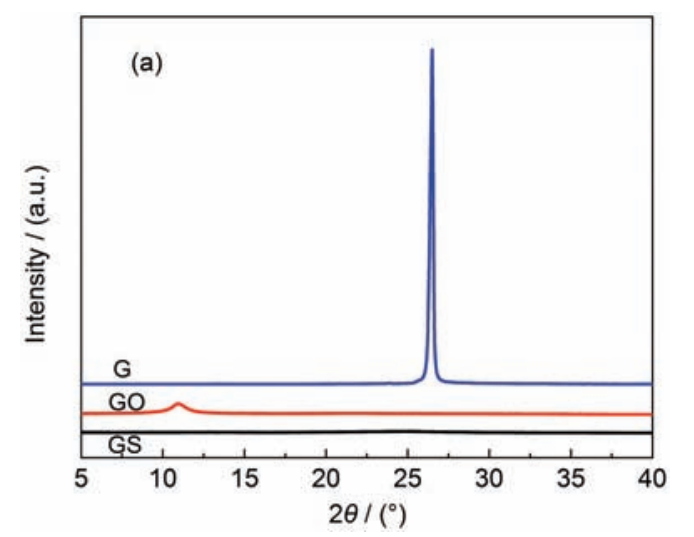

节温度控制器, 设定吸附温度分别为 25 和 $55^{\circ} \mathrm{C}$, 在 100-2500 kPa 压力范围内取不同的压力点, 吸附仪 记录了样品重量随设置压力点的变化曲线, 通过数 据处理, 即可得到在不同温度条件下的氢气吸附等 温线.

\section{3 结果与讨论}

\section{$3.1 X$ 射线衍射分析}

图 1 是石墨 $(\mathrm{G})$ 、氧化石墨 $(\mathrm{GO})$ 、石墨烯 $\left(\mathrm{GS}_{1}\right.$ 、 $\mathrm{GS}_{2}$ ) 的 XRD 图. 从图 1(a)中可以看出, 石墨在 $2 \theta$ 为 $26.58^{\circ}$ 处的(002)晶面衍射峰很强很尖, 说明石墨片 层的空间排列非常规整. 氧化石墨在 $2 \theta$ 为 $10.60^{\circ}$ 处 的(001)晶面衍射峰 ${ }^{28}$ 很强, 而石墨(002)面的衍射峰 变得很小, 这说明石墨晶型结构被破坏, 生成了新 的氧化石墨晶体结构. 当氧化石墨被还原成石墨烯 后, 在 $2 \theta$ 为 $26.08^{\circ}$ 处出现衍射峰, 和石墨的衍射峰相 比, 衍射峰变宽, 强度减弱. 这是由于石墨烯相对石 墨而言片层更小, 无序度增加. 而图 1(b)中石墨烯 $\mathrm{GS}_{1}$ 比 $\mathrm{GS}_{2}$ 的衍射峰明显微弱得多, 这是由于分散剂 的活性作用阻碍了石墨烯片层间团聚的发生, 制备 的石墨烯 $\mathrm{GS}_{1}$ 大多都是单层结构, 其层间距远大于 $\mathrm{X}$ 射线的波长, 从而导致衍射现象不明显. ${ }^{29}$ 这也说 明添加分散剂可以作为制备单层石墨烯的一种良 好的方法.

\subsection{SEM 和 TEM 分析}

图 2 是石墨烯 $\mathrm{GS}_{1}$ 和 $\mathrm{GS}_{2}$ 的 $\operatorname{SEM}(\mathrm{a}, \mathrm{b})$ 和 TEM (c, d) 照片. 从图 2(a) 可以看出, 石墨烯 $\mathrm{GS}_{1}$ 是由一层 层蜂窝状的单片结构组成, 排列较规整, 孔道结构 明显, 表明在还原过程中很少有团聚现象. 而图 2(b) 中石墨烯 $\mathrm{GS}_{2}$ 单片堆叠在一起, 排列紧密, 表明在还

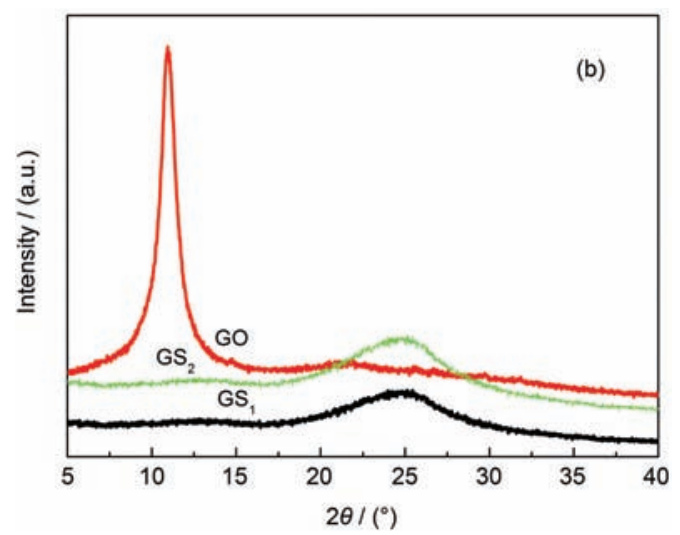

图 $1 \mathrm{G} 、 G O 、 G_{1} 、 G S_{2}$ 的 XRD 图谱(a)及其放大图谱(b)

Fig.1 XRD patterns (a) and their magnified patterns (b) of G, GO, GS 1 , and $\mathrm{GS}_{2}$

$\mathrm{G}$ : graphite, GO: graphite oxide, $\mathrm{GS}_{1}$ : graphene with surfactant, $\mathrm{GS}_{2}$ : graphene without surfactant 

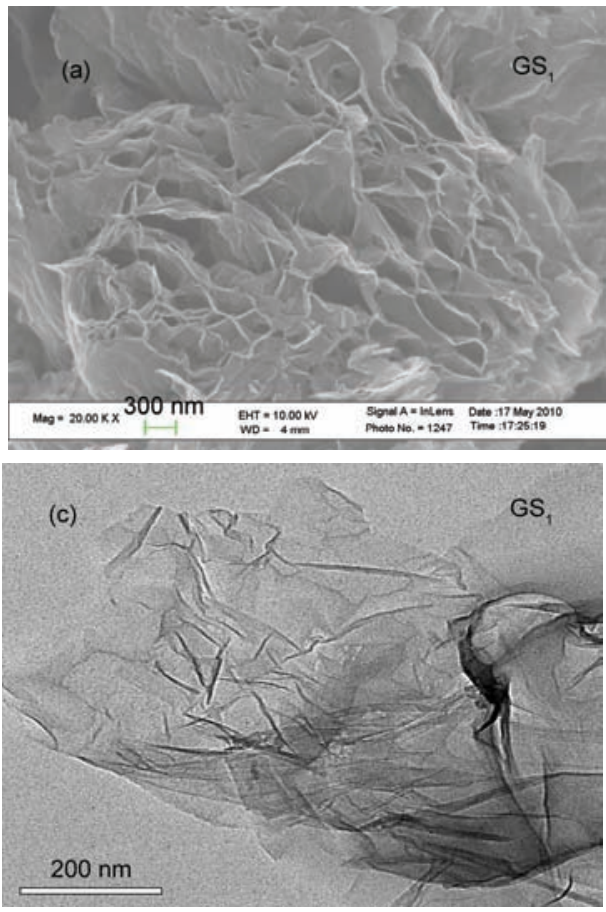

图 2 石墨烯 $\mathrm{GS}_{1}$ 和 $\mathrm{GS}_{2}$ 的 SEM 图(a,b)和 TEM 图(c,d)

Fig.2 SEM $(a, b)$ and TEM $(c, d)$ images of $G_{1}$ and $G_{2} S_{2}$

原过程中仍然有大量团聚现象发生. 这是由于分散 剂的作用使 $\mathrm{GS}_{1}$ 的悬浮液分散效果明显优于 $\mathrm{GS}_{2}$. 从 图 2(c) 可以看出石墨烯 $\mathrm{GS}_{1}$ 是几乎透明的薄纱状结 构, 表面平整、规则, 缺陷较少, 说明 $\mathrm{GS}_{1}$ 含有很少层 数的石墨烯. 而图 2(d)中石墨烯 $\mathrm{GS}_{2}$ 表面不平整, 厚 度不均一, 表明 $\mathrm{GS}_{2}$ 有大量单层石墨烯重叠在一起, 发生卷曲. 以上结果表明, 通过分散剂辅助还原氧 化石墨的方法可制备出高质量石墨烯样品 $\mathrm{GS}_{1}$.

通过以上 XRD、SEM 和 TEM 分析, 可以看出石 墨烯 $\mathrm{GS}_{1}$ 的晶型、形貌结构和分散效果均明显优于 $\mathrm{GS}_{2}$, 因此, 在后续表征中重点对 $\mathrm{GS}_{1}$ 进行拉曼和 AFM 分析.

\section{3 拉曼光谱分析}

图 3 是石墨 $\mathrm{G}$ 、氧化石墨 $\mathrm{GO}$ 和石墨烯 $\mathrm{GS}_{1}$ 的拉 曼光谱. 由图可知, 石墨在 $1576 \mathrm{~cm}^{-1}$ 处有一个尖而 强的吸收峰 $\left(G\right.$ 峰), 而在 $1332 \mathrm{~cm}^{-1}$ 处有一个很弱的 吸收峰 ( $D$ 峰), 说明石墨的 $s p^{2}$ 杂化碳原子结构排列 非常紧密规整. 在石墨被氧化后, 氧化石墨的 $G$ 峰 变宽, 且蓝移至 $1578 \mathrm{~cm}^{-1}$, 而 $D$ 峰强度也增加, 这表 明石墨被氧化后, 结构中一部分 $s p^{2}$ 杂化碳原子转化 成 $s p^{3}$ 杂化结构, 即石墨中的 $\mathrm{C}=\mathrm{C}$ 双键被破坏. 此 外, $D$ 带与 $G$ 带的强度比 $\left(I_{D} / I_{G}\right)$ 也表示 $s p^{3} / s p^{2}$ 碳原子 比, ${ }^{30}$ 也表明氧化石墨中 $s p^{2}$ 杂化碳层平面长度比石 墨小. 当氧化石墨被还原后, $D$ 峰和 $G$ 峰的峰位和石
墨的相似, 说明氧化石墨中含氧官能团被大量去 除, 而石墨烯的 $D$ 峰和 $G$ 峰两个峰的强度比 $\left(I_{D} / I_{G}=\right.$ 1.28) 高于氧化石墨的两个峰的强度比 $\left(I_{D} / I_{G}=1.06\right)$, 这说明石墨烯中 $s p^{2}$ 杂化碳层平面的平均尺寸比氧 化石墨的大,$^{31}$ 并且石墨烯片层无序度增加. 同时也 证明氧化石墨被还原后, 只有一部分 $s p^{3}$ 杂化碳原子 被还原成 $s p^{2}$ 杂化碳原子, 即氧化石墨的结构不可能 完全恢复为石墨结构, 石墨烯和石墨的结构存在一 定区别.

\subsection{AFM 分析}

图 4 为石墨烯 $\mathrm{GS}_{1}$ 的 $\mathrm{AFM}$ 分析照片. 由图可以 看出石墨烯纳米片均匀地分散在云母片基底上, 呈

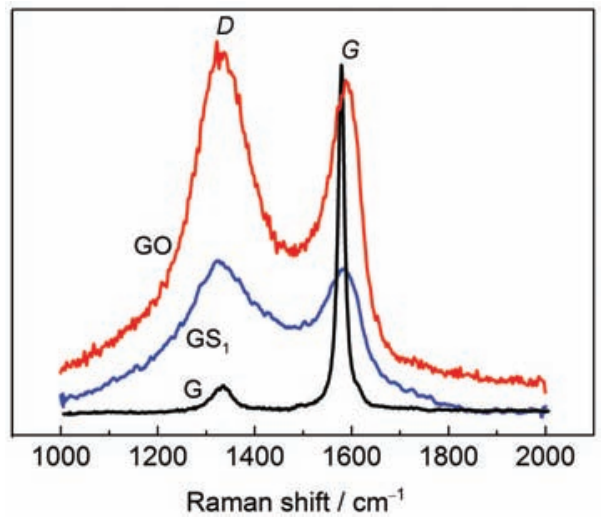

图 $3 \mathbf{G} 、 \mathbf{G O}$ 和 $\mathbf{G S}_{1}$ 的拉曼谱图

Fig.3 Raman spectra of G, GO, and GS $_{1}$ 


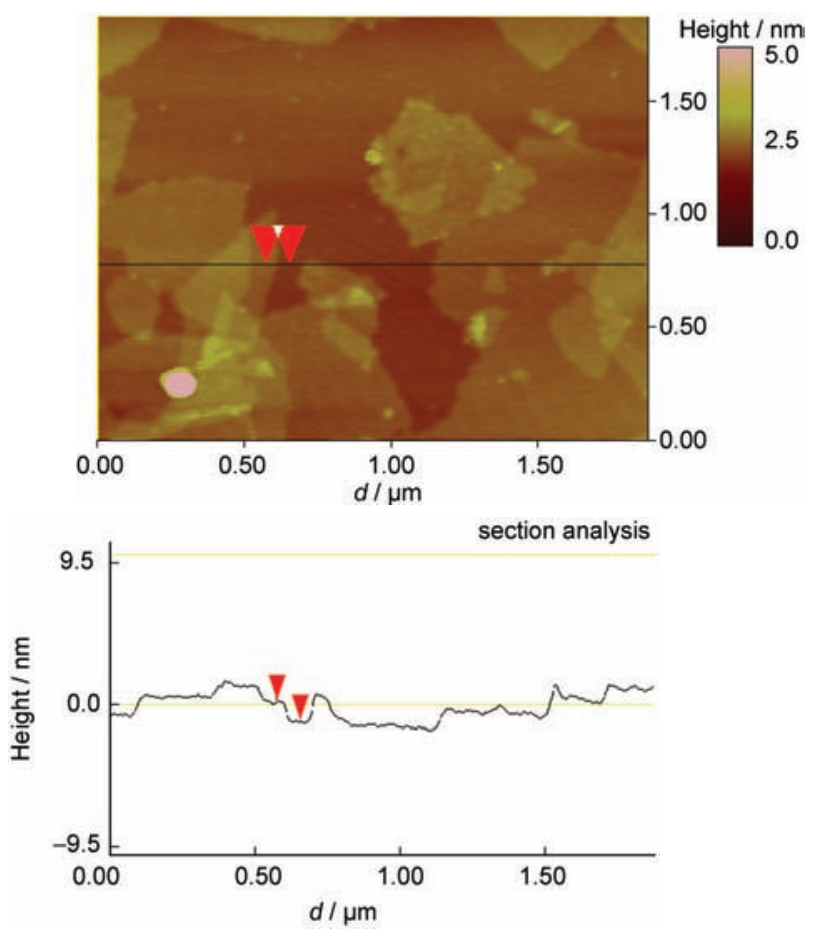

图 4 石墨烯 $\mathrm{GS}_{1}$ 的 AFM 图

Fig.4 AFM images of GS $_{1}$

少量堆叠状态分布, 样品颜色均一, 和基底颜色区 分明显. Stankovich 等 ${ }^{24,32}$ 已经从理论和实验证明, 液 相化学还原法制得的单层石墨烯的厚度约为 1.1 $\mathrm{nm}$. 根据 AFM 的分析结果, 石墨烯样品 $\mathrm{GS}_{1}$ 的厚度 约为 $1.3 \mathrm{~nm}$, 表明所制备的石墨烯样品基本上为单 层, 也可能存在一些双层或三层, 再次证明了添加 分散剂辅助还原氧化石墨可以得到高质量的石墨 烯.

\subsection{BET 比表面积和孔径分布}

图 5 和图 6 分别给出了石墨烯样品 $\mathrm{GS}_{1}$ 和 $\mathrm{GS}_{2}$ 在 液氮温度下的 $\mathrm{N}_{2}$ 吸附-脱附等温线和孔径分布. 从 图 5 可知, 整个 $\mathrm{N}_{2}$ 吸附等温线呈 I 型吸附曲线, 在相

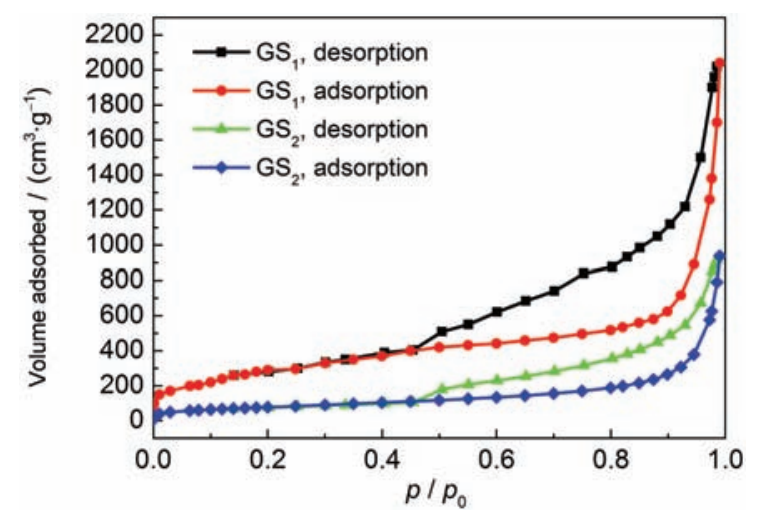

图 5 石墨烯 $G_{1} S_{1}$ 和 $G S_{2}$ 的 $N_{2}$ 吸附-脱附等温线

Fig.5 $\mathrm{N}_{2}$ adsorption-desorption isotherms of $\mathrm{GS}_{1}$ and $\mathrm{GS}_{2}$

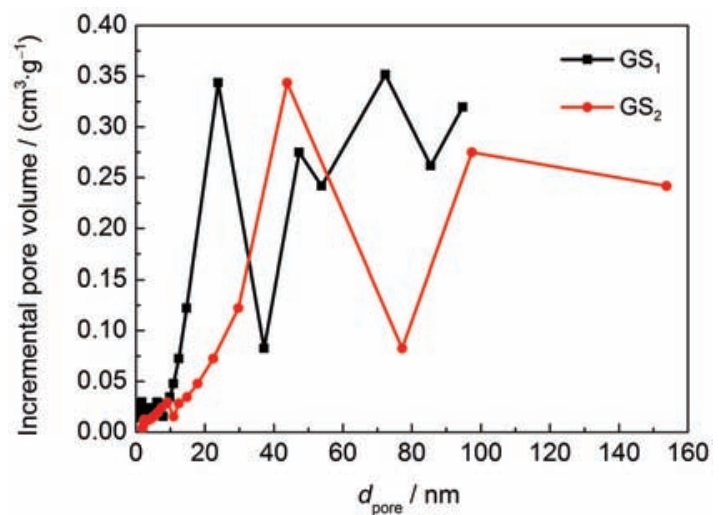

图 6 石墨烯 $\mathrm{GS}_{1}$ 和 $\mathrm{GS}_{2}$ 的孔径分布图

Fig.6 Pore size distributions of $\mathrm{GS}_{1}$ and $\mathrm{GS}_{2}$

对压力约为 0.4 时等温吸附-脱附曲线上出现磁滞 环, 表明石墨烯层间存在狭长的缝隙 ${ }^{3}$ 经过 BET 比 表面积分析可得石墨烯样品 $\mathrm{GS}_{1}$ 比表面积约为 $1206 \mathrm{~m}^{2} \cdot \mathrm{g}^{-1}$, 远远大于 $\mathrm{GS}_{2}$ 的比表面积 $605 \mathrm{~m}^{2} \cdot \mathrm{g}^{-1}$, 而小于单层石墨烯的理论比表面积 $\left(2630 \mathrm{~m}^{2} \cdot \mathrm{g}^{-1}\right)$, 这说明石墨烯片 $\mathrm{GS}_{1}$ 并非完全由单层石墨烯组成, 而是存在少许双层结构, 但相比之前研究有了很大 突破, 详见表 1. 图 6 的孔径分布表明石墨烯 $\mathrm{GS}_{1}$ 的 孔径分布范围小于 $\mathrm{GS}_{2}$, 含有微孔的数量也大于 $\mathrm{GS}_{2}$, 这进一步说明了 $\mathrm{GS}_{1}$ 的比表面积大于 $\mathrm{GS}_{2}$ 和之 前研究成果的原因.

\section{4 氢气的高压吸附性能评价}

图 7 为本研究所制备的石墨烯 $\mathrm{GS}_{1}$ 和 $\mathrm{GS}_{2}$ 在压 力范围 100-2500 kPa, 温度为 $25^{\circ} \mathrm{C}$ 和 $55^{\circ} \mathrm{C}$ 条件下 氢气高压吸附实验结果比较(图中标明了氢气吸附 量的平均值和标准偏差).

由图 7 可见, 石墨烯 $\mathrm{GS}_{1}$ 的氢气吸附量明显大

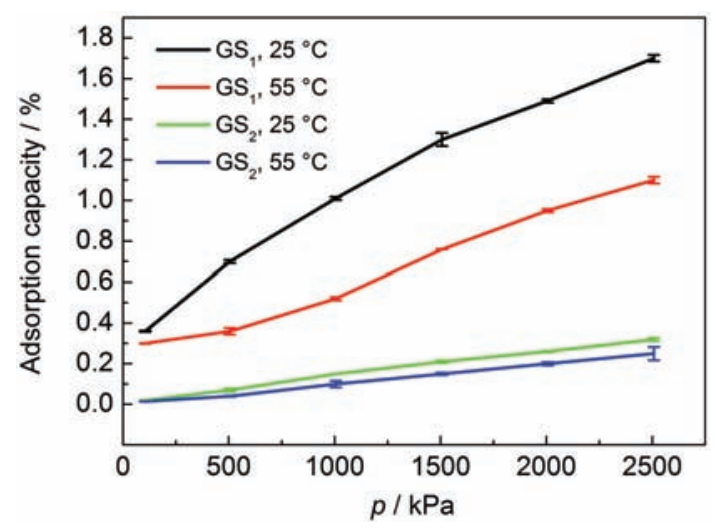

图 725 和 $55^{\circ} \mathrm{C}$ 时不同压力下石墨烯 $\mathrm{GS}_{1}$ 和 $\mathrm{GS}_{2}$ 的 氢气吸附量

Fig.7 $\mathrm{H}_{2}$ adsorption capacity of $\mathrm{GS}_{1}$ and $\mathrm{GS}_{2}$ under different pressures at 25 and $55^{\circ} \mathrm{C}$ 
表 $125^{\circ} \mathrm{C}$ 时不同方法制备石墨烯的比表面积和氢气吸附量

Table 1 BET surface area and $\mathrm{H}_{2}$ adsorption capacity of graphene with different preparation methods $25^{\circ} \mathrm{C}$

\begin{tabular}{cccc}
\hline Prepared method & $S /\left(\mathrm{m}^{2} \cdot \mathrm{g}^{-1}\right)$ & $\Gamma_{\mathrm{H}_{2}} / \%(w)$ & Reference \\
\hline liquid oxidation-reduction & 640 & 0.1 & 20 \\
thermal exfoliation & 156 & $<0.2$ & 22 \\
liquid oxidation-reduction & 466 & - & 24 \\
$\begin{array}{c}\text { liquid oxidation-reduction } \\
\text { (without surfactant) }\end{array}$ & 605 & 0.3 & - \\
$\begin{array}{c}\text { liquid oxidation-reduction } \\
\text { (with surfactant) }\end{array}$ & 1206 & 1.7 & - \\
\hline
\end{tabular}

于 $\mathrm{GS}_{2}$ 的, 在 25 和 $55^{\circ} \mathrm{C}$ 下, 对于不同的压力, 石墨 烯 $\mathrm{GS}_{1}$ 和 $\mathrm{GS}_{2}$ 对氢气的吸附量各不相同. 在同一温度 下, 随着压力的增大, 石墨烯的氢气吸附量逐渐增 大; 在相同的压力下, 石墨烯对氢气的吸附量随着 温度的增加而减小, 这种现象符合物理吸附的特 点. ${ }^{34}$ 在氢气压力相对较低时, 氢气分子优先吸附于 微孔内, 发生微孔吸附, 氢气吸附量相对较低, 但 $\mathrm{GS}_{1}$ 含有的微孔数大于 $\mathrm{GS}_{2}$, 在吸附的初始阶段 $\mathrm{GS}_{1}$ 的氢气吸附量大于 $\mathrm{GS}_{2}$. 随着压力的增大, 吸附逐渐 从中孔、大孔转移至石墨烯外表面, 发生外表面吸 附, 同时, 氢气吸附量也相应增大. 在温度 $25^{\circ} \mathrm{C}$, 压 力 $2500 \mathrm{kPa}$ 的条件下, $\mathrm{GS}_{1}$ 的氢气吸附量高达 $1.7 \%$ $(w)$, 远大于同类方法得到的研究结果, 如表 1 所示.

由表 1 可见, 与氧化还原过程不加分散剂的方 法制备的石墨烯片相比, 添加分散剂后, 石墨烯片 的比表面积和氢气吸附量都有明显的增加.

石墨烯在温度 $T_{1}$ 和 $T_{2}$ 下对氢气的吸附热由克 劳修斯-克拉伯龙方程(1)计算得知约为 $18.6 \mathrm{~kJ}$. $\mathrm{mol}^{-1}$, 这个结果和美国能源部提出的最理想的吸附 材料的吸附热值 $\left(15 \mathrm{~kJ} \cdot \mathrm{mol}^{-1}\right)^{35,36}$ 很接近, 进一步证 明了通过改进液相氧化还原法所制备的石墨烯是 一种良好的氢气吸附材料.

$$
Q_{\text {st }}=\frac{R \cdot \ln \left(p_{T_{1}} / p_{T_{2}}\right)}{\left(1 / T_{1}-1 / T_{2}\right)}
$$

其中, $Q_{\mathrm{st}}$ 为吸附热 $\left(\mathrm{kJ} \cdot \mathrm{mol}^{-1}\right), R$ 为真实气体常数 $\left(8.314 \mathrm{~kJ} \cdot \mathrm{mol}^{-1} \cdot \mathrm{K}^{-1}\right), p_{T_{1}}$ 和 $p_{T_{2}}$ 分别为温度 $T_{1}$ 和 $T_{2}$ 相对应的氢气压力 $(\mathrm{kPa})$.

\section{5 结 论}

通过液相氧化法制备氧化石墨, 超声剥离后, 在还原过程中添加分散剂十二烷基苯磺酸钠制备 了高质量的石墨烯片. 采用 XRD、拉曼光谱、SEM、 $\mathrm{TEM}$ 和 $\mathrm{AFM}$ 等手段表征了其结构、形貌和组成, 结
果表明所制备的石墨烯片基本上为单层，也存在少 量的双层结构, BET 结果表明石墨烯片含有较大的 比表面积和丰富的孔状结构. 氢气的高压吸附实验 表明在温度为 $25^{\circ} \mathrm{C}$, 压力为 $2500 \mathrm{kPa}$ 条件下, 石墨 烯对氢气吸附量高达 $1.7 \%(w)$, 证明了本改进液相 氧化还原方法制备石墨烯可有效减小石墨烯单层 之间的团聚, 从而增加石墨烯的比表面积, 提高氢 气吸附量, 同时也体现了石墨烯在绿色氢能方面广 阔的应用前景.

\section{References}

(1) Haryanto, A.; Fernando, S.; Murali, N.; Adhikari, S. Energy and Fuels 2005, 19 (5), 2098.

(2) Barelli, L.; Bidini, G.; Gallorini, F.; Servili, S. Energy 2008, 33 (4), 554.

(3) Rosen, M. A. Energy 2010, 35, 1068.

(4) Dillon, A. C.; Jones, K. M.; Bekkedahl, T. A.; Kiang, C. H.; Bethune, D. S.; Heben, M. J. Nature 1997, 386, 377.

(5) Liu, C.; Fan, Y. Y.; Liu, M.; Cong, H. T.; Cheng, H. M.; Dresselhaus, M. S. Science 1999, 286, 1127.

(6) Zhou, L.; Zhou, Y. P.; Sun, Y. Int. J. Hydrog. Energy 2004, 29 (5), 475.

(7) Fan, Y. Y.; Liao, B.; Liu, M.; Wei, Y. L.; Lu, M. Q.; Cheng, H. M. Carbon 1999, 37, 1649 .

(8) Gupta, B. K.; Srivastava, O. N. Int. J. Hydrog. Energy 2001, 26 , 857.

(9) Zhou, L.; Zhou, Y. P.; Sun, Y. Int. J. Hydrog. Energy 2004, 29 (3), 319.

(10) Huang, G. R.; Chen, J. Carbon Technologies 2009, 1 (28), 35

(11) Novoselov, K. S.; Geim, A. K.; Morozov, S. V.; Jiang, D.; Zhang, Y.; Dubonos, S. V.; Grigorieva, I. V.; Firsov, A. A. Science 2004, 306, 666 .

(12) Allen, M. J.; Tung, V. C.; Kaner, R. B. Chem. Rev. 2010, 110, 132.

(13) Stoller, M. D.; Park, S.; Zhu, Y.; An, J.; Ruoff, R. S. Nano Lett. 2008, 8 (10), 3498.

(14) Yang, N.; Zhai, J.; Wang, D.; Chen, Y.; Jiang, L. ACS Nano 2010, 4, 887 .

(15) Hu, Y. J.; Jin, J.; Zhang, H.; Wu, P.; Cai, C. X. Acta Phys. -Chim. Sin. 2010, $26(8), 2073$. [胡耀娟, 金 娟, 张 卉, 吴 萍, 蔡称心. 物理化学学报, 2010, 26 (8), 2073.]

(16) Sridhar, V.; Jeon, J. H.; Oh, I. K. Carbon 2010, 48(10), 2953.

(17) Wen, Z. L.; Yang, S. D.; Song, Q. J.; Hao, L.; Zhang, X. G. Acta Phys. -Chim. Sin. 2010, 26 (6), 1570. [温祝亮, 杨苏东, 宋启 军, 郝 亮, 张校刚. 物理化学学报, 2010, 26 (6), 1570.]

(18) Wu, X. Q.; Zong, R. L.; Mou, H. J.; Zhu, Y. F. Acta Phys. -Chim. Sin. 2010, 26 (11), 3002. [吴小琴, 宗瑞隆, 牟豪杰, 朱永法. 物理化学学报, 2010, 26 (11), 3002.]

(19) Li, Y. X.; Wei, Z. D.; Zhao, Q. L.; Ding, W.; Zhang, Q.; Chen, S. 
G. Acta Phys. -Chim. Sin. 2011, 27 (4), 858. [李云霞, 魏子栋, 赵巧玲, 丁 炜, 张 寒, 陈四国. 物理化学学报, 2011, 27 (4), 858.]

(20) Srinivas, G.; Zhu, Y. W.; Piner, R.; Skipper, R.; Ellerby, M.; Ruoff, R. Carbon 2010, 48 (3), 630.

(21) Ghosh, A.; Subrahmanyam, K. S.; Krishna, K. S.; Datta, S.; Govindaraj, A.; Pati, S. K.; Rao, C. N. R. J. Phys. Chem. C 2008, $112,15704$.

(22) Ma, L. P.; Wu, Z. S.; Li, J.; Wu, E. D.; Ren, W. C.; Cheng, H. M. Hydrogen Energy 2009, 34 (5), 2329.

(23) Bourlinos, A. B.; Gournis, D.; Petridis, D.; Szabó, T.; Szeri, A.; Dékány, I. Langmuir 2003, 19, 6050.

(24) Stankovich, S.; Dikin, D. A.; Piner, R. D.; Kohlhaas, K. A.; Kleinhammes, A.; Jia, Y.; Wu, Y.; Nguyen, S. T.; Ruoff, R. S. Carbon 2007, 45, 1558

(25) Lotya, M.; King, P. J.; Khan, U.; De, S.; Coleman, J. N. ACS Nano 2010, 4 (6), 3155.

(26) Xu, Y. X.; Bai, H.; Lu, G. W.; Li, C.; Shi, G. Q. J. Am. Chem.
Soc. 2008, 130 (18), 5856.

(27) Hummers, S.; Offeman, R. J. Am. Chem. Soc. 1958, 80 (6), 1339.

(28) Wang, J.; Han, Z. D. Polym. Adv. Technol. 2006, 17 (4), 335.

(29) Lee, S.; Lim, S.; Lim, E.; Lee, K. K. Journal of Physics and Chemistry of Solids 2010, 71 (4), 483.

(30) Ferrari, A. C.; Robertson, J. J. Phys. Rev. B 2000, 61 (20), 14095.

(31) Gomez-Navarro, C.; Weitz, R. T.; Bittner, A. M.; Scolari, M.; Mews, A.; Burghard, M.; Kern, K. Nano Lett. 2007, 7 (11), 3499.

(32) Jung, I.; Pelton, M.; Piner, R.; Dikin, D.A.; Stankovich, S.; Watcharotone, S. Nano. Lett. 2007, 7 (12), 3569.

(33) Szabo, T.; Berkesi, O.; Dekany, I. Carbon 2005, 43 (15), 3186.

(34) Jhi, S. H.; Kwon, Y. K.; Bradley, K. P.; Gabriel, J. C. Solid State Communications 2004, 129, 769.

(35) Gigras, A.; Bhatia, S. K.; Anil Kumar, A.V.; Myers, A. L. Carbon 2007, 45 (5), 1043.

(36) Bhatia, S. K.; Myers, A. L. Langmuir 2006, 22 (4), 1688. 